\title{
INCREASED ALDOSTERONE SECRETION IN DOGS WITH RIGHT- SIDED CONGESTIVE HEART FAILURE AND IN DOGS WITH THORACIC INFERIOR VENA CAVA CONSTRICTION
}

\author{
By JAMES O. DAVIS, MAURICE M. PECHET, WILMOT C. BALL, JR, AND \\ M. JAY GOODKIND 1 wITH THE SURGICAL ASSISTANCE OF ALFRED CASPER \\ (From the Laboratory of Kidney and Electrolyte Metabolism, National Heart Institute, \\ National Institutes of Health, USPHS, Bethesda, Maryland, and the \\ Massachusetts General Hospital, Boston, Mass.)
}

(Submitted for publication October 10, 1956; accepted January 24, 1957)

Studies (1-5) of patients with congestive heart failure, decompensated hepatic cirrhosis and nephrosis have provided evidence of increased amounts of aldosterone or aldosterone-like substances in urine during edema or ascites formation. More recently, it has been demonstrated (6) that increased urinary excretion of aldosterone occurs in association with sodium retention in dogs with right heart failure and in dogs with ascites secondary to constriction of the thoracic inferior vena cava. The data in both man and dog imply that the blood level of aldosterone is elevated.

Numerous attempts have been made to elucidate the mechanism regulating aldosterone secretion in clinical states associated with the formation of edema, but no data have been reported to show that increased circulating aldosterone results from increased secretion rather than from a decreased rate of inactivation. The purpose of this study was to determine the relative rates of secretion of aldosterone in 1) dogs with cardiac failure produced by stenosis of the pulmonary artery, 2) dogs with thoracic inferior vena cava constriction and ascites, and 3 ) normal dogs.

\section{METHODS}

Right heart failure was produced in four dogs by controlled progressive constriction of the main pulmonary artery by a method described previously (7). In three other animals, ascites formed following constriction of the thoracic inferior vena cava. Six normal dogs provided the control material. All animals were female mongrel dogs weighing 15 to $23 \mathrm{~kg}$. The dogs were fed a synthetic diet containing $60 \mathrm{mEq}$. of sodium and $18 \mathrm{mEq}$. of potassium per day with two exceptions; one dog with heart failure and one normal dog received $40 \mathrm{mEq}$. per day of sodium and $40 \mathrm{mEq}$. per day of potassium. At the

\footnotetext{
1 Present address: First Medical Division, Bellevue Hospital, New York, N. Y.
}

time adrenal vein blood was obtained, all dogs with heart failure or caval constriction had a high venous pressure; ascites was present and urinary sodium excretion was less than $10 \mathrm{mEq}$. per day.

Adrenal vein blood was obtained under two sets of circumstances. First, blood was collected with the animals under sodium pentobarbital anesthesia; no infusion was given during the procedure. In the second group of animals, adrenal vein blood was collected with the dogs awake. In this group, a local anesthetic (Xylocaine) was used for the skin and muscle of the abdominal wall but none was injected near the adrenal gland; morphine sulfate (5 to $10 \mathrm{mgm}$.) was given subcutaneously to all but one dog in the second group. This series of dogs received a blood transfusion at approximately the same rate that adrenal venous blood was obtained. Peripheral blood was collected from two of the dogs of the second group. The peripheral blood was obtained from the dog with cardiac failure immediately after collection of adrenal vein blood and from the dog with thoracic caval constriction on the day before collection of adrenal vein blood.

Adrenal venous blood (300 cc.) was collected by cannulating the right. adrenolumbar vein with a polyethylene catheter located peripheral to the point of venous inflow from the right adrenal gland. All tributaries to the adrenolumbar vein in this region other than the adrenal veins were ligated and the adrenolumbar vein was occluded at its junction with the inferior vena cava. By this procedure, flow of venous blood from the right adrenal gland was diverted from its normal pathway into the polyethylene catheter. Heparin (40 to $50 \mathrm{mgm}$.) was injected intravenously into each animal before collection of blood was begun. Flow was measured by collecting blood in a graduated cylinder. The cylinder was immersed in an ice bath and each 25 cc. collection was transferred immediately to a flask of acetone surrounded by ice.

The procedure for isolation of aldosterone from adrenal venous and peripheral blood is shown in Figure 1. An aqueous acetone extract of the blood was concentrated by evaporation in an atmosphere of nitrogen and partitioned twice between petroleum ether and aqueous 70 per cent ethanol. The aqueous ethanol extract was similarly concentrated and then extracted with methylene chloride. This methylene chloride extract was concentrated and 
subjected to column partition chromatography, with ethylene glycol as the stationary phase and mixtures of methylene chloride and cyclohexane as the mobile phase. No attempt was made to remove compound $E$ from the aldosterone fraction since compound $\mathrm{E}$ does not interfere with the assay for aldosterone (8) unless the amount present far exceeds that found in adrenal vein blood from dogs (9). Also, the combination of compound $E$ and aldosterone produces less $\mathrm{Na}$ retention by assay than does aldosterone alone (8).

The various chromatographic fractions of adrenal vein and peripheral blood were assayed in bilaterally adrenalectomized female dogs according to the assay procedure of Liddle, Cornfield, Casper, and Bartter (8) except that the crossover design was not employed. The adrenalectomized dogs were maintained on a diet containing 200 mEq. per day of sodium and $18 \mathrm{mEq}$. per day of potassium; $5 \mathrm{mgm}$. per day of cortisone acetate were given intramuscularly 18 hours before the assay. Sodiumretaining and potassium-excreting activity obtained by assay was expressed in terms of aldosterone or aldosterone equivalents which were determined from a doseresponse curve obtained previously (6).

\section{RESULTS}

A comparison of the concentration of aldosterone in peripheral and adrenal vein blood is presented in Figure 2. Adrenal vein blood from the dog with cardiac failure contained $12.3 \mu \mathrm{g}$. in 300 cc. while blood from the dog with thoracic

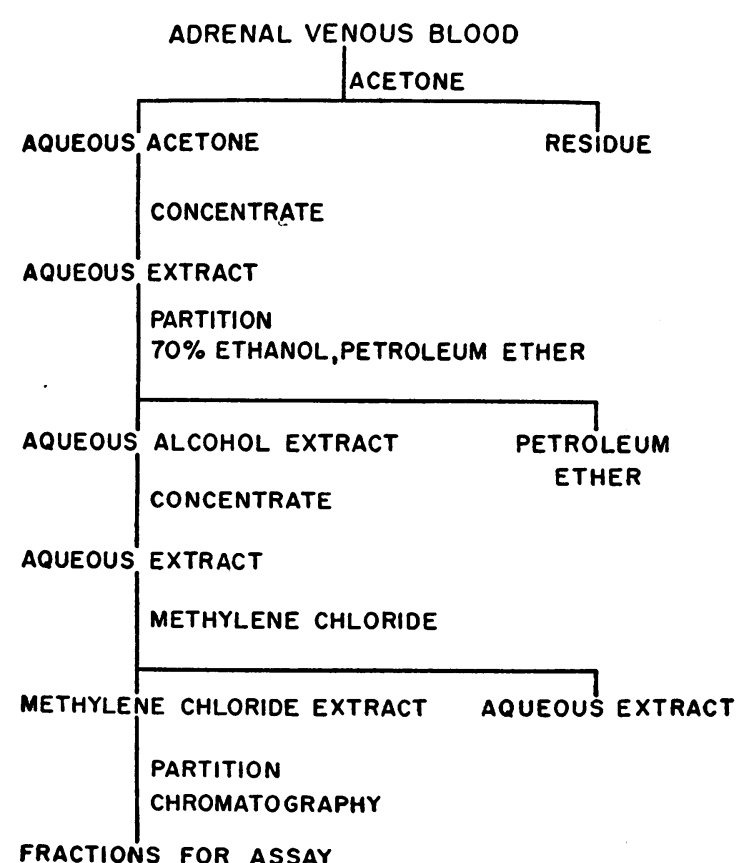

Fig. 1. Method of Extraction of Adrenal Vein and Peripheral Blood caval constriction showed $5.0 \mu \mathrm{g}$. in $300 \mathrm{cc}$. No aldosterone was detected in the same quantity of peripheral blood from either dog. The data demonstrate that the aldosterone present in adrenal venous effluents was secreted aldosterone rather than circulating hormone.

Studies of aldosterone production demonstrated a higher rate of secretion in dogs with thoracic caval constriction or heart failure than in normal dogs (Table I). The initial observations were made using sodium pentobarbital anesthesia to obtain adrenal vein blood. In normal animals, aldosterone was not detected in 300 cc. of adrenal vein blood; the assay values were less than 4.0 $\mu \mathrm{g}$. of aldosterone equivalents and were of the same order of magnitude for all chromatographic fractions. In contrast, aldosterone was present in concentrations of 9.0 and $15.3 \mu \mathrm{g}$. per $300 \mathrm{cc}$. of adrenal vein blood from the dog with thoracic caval constriction and from the dog with heart failure. The rates of aldosterone secretion were 6.0 and $4.9 \mu \mathrm{g}$. per hr., respectively, for these two animals.

Observations on aldosterone secretion using local anesthesia also showed a higher rate of secretion in dogs with caval constriction or heart failure than in normal dogs (Table I). In addition, the rates of aldosterone production were higher during local anesthesia in both normal dogs and dogs with heart failure or caval constriction than

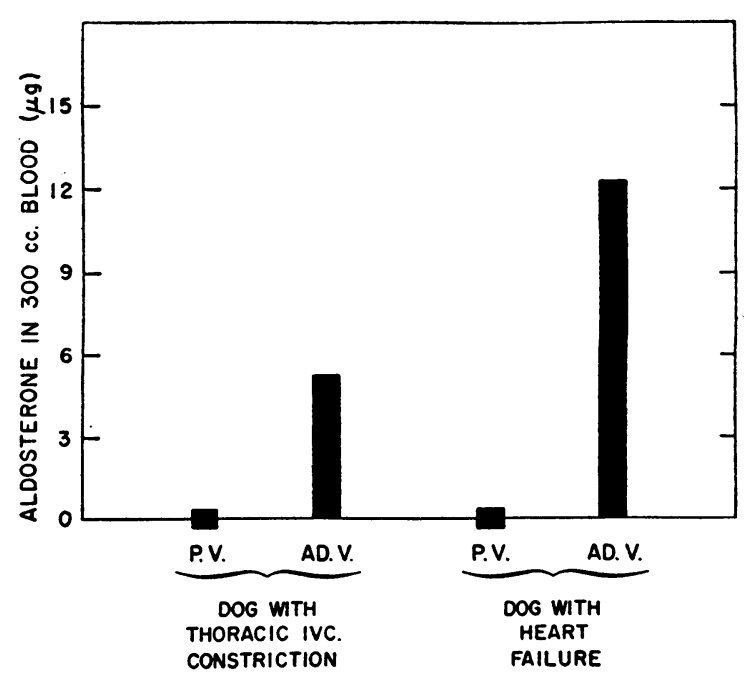

Fig. 2. Comparison of the Aldosterone Content of Peripheral Vein (P.V.) and Adrenal Vein (AD.V.) BLOOD 
TABLE I

Results from assay of chromatographic fractions of adrenal vein blood and measurements of adrenal blood flow, aldosterone secretion and venous pressure

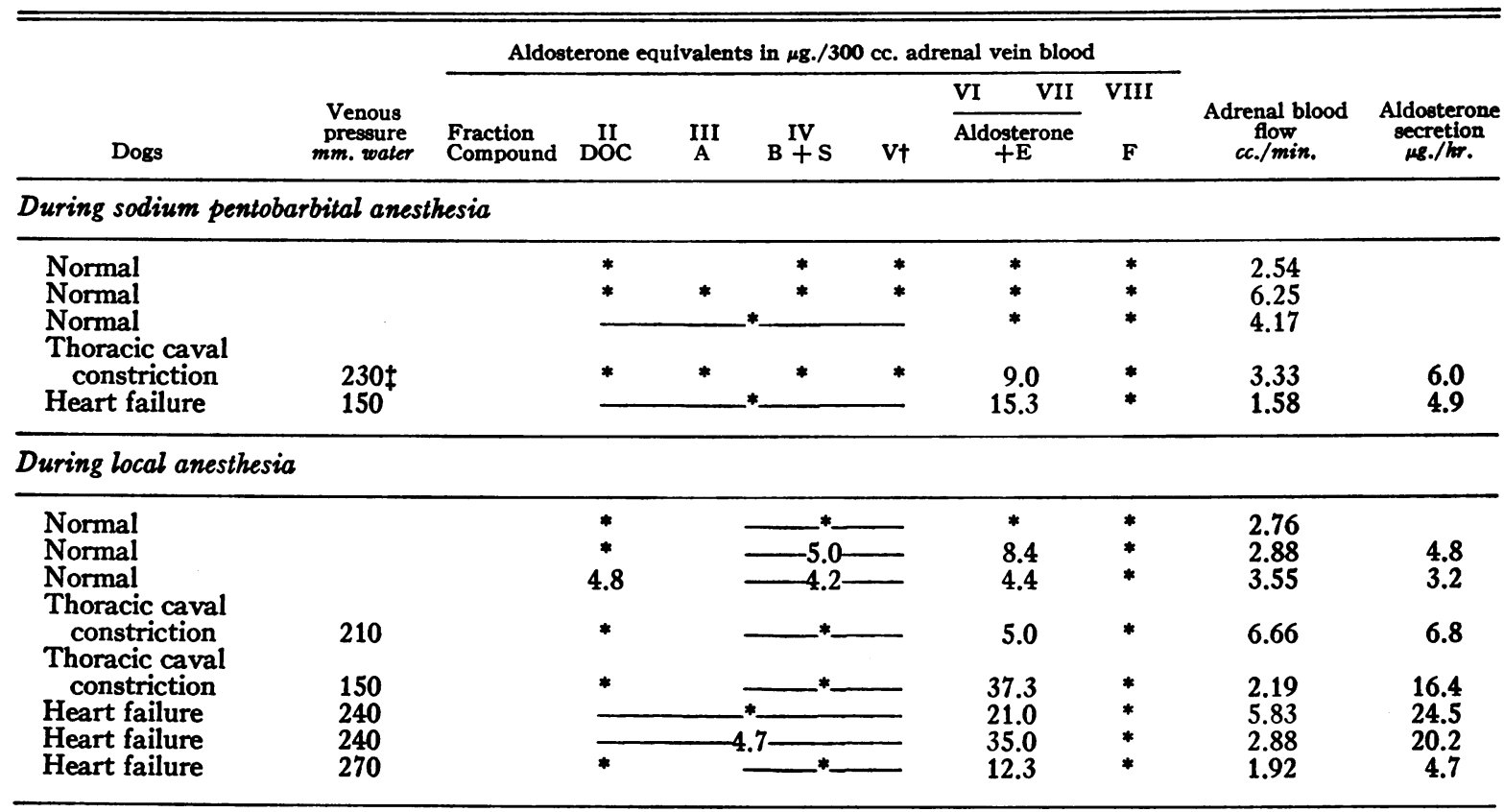

* Indicates that biological activity by assay was less than $4.0 \mu \mathrm{g}$. of aldosterone equivalents per $300 \mathrm{cc}$. of adrenal vein blood; $4.0 \mu \mathrm{g}$. is the smallest amount of activity detectable by this assay procedure.

f Fraction V was obtained by using the same mixture of methylene chloride and cyclohexane as for Fraction IV.

¥ Values for venous pressure were obtained at the beginning of the collection of adrenal vein blood. The normal range of venous pressure is 35 to $70 \mathrm{~mm}$. water (7).

during sodium pentobarbital anesthesia. Aldosterone was found in adrenal vein blood collected under local anesthesia from two of three normal dogs and the rates of secretion were 3.2 and 4.8 $\mu \mathrm{g}$. per hr. No aldosterone was detected in adrenal vein blood from the other normal dog. These findings in normal dogs are in contrast with the high rates of aldosterone secretion of 6.8 and $16.4 \mu \mathrm{g}$. per hr. for the dogs with caval constriction and of 4.7, 20.2 and $24.5 \mu \mathrm{g}$. per hr. for the dogs with heart failure. Statistical comparison of the rate of aldosterone secretion in 6 normal dogs with that in the 7 dogs with either caval constriction or heart failure revealed a significant difference $(\mathrm{T}=3.0 ; \mathrm{P}<0.02)$.

The results from assay of fractions of adrenal vein blood other than the aldosterone fraction showed no consistent evidence of activity causing sodium retention or increased potassium excretion for any fraction. It should be pointed out also that three of the four non-aldosterone fractions which showed activity were from adrenal vein blood from normal dogs. Consequently, the data demonstrate that increased biological activity of this type in adrenal vein blood from dogs with heart failure and from dogs with caval constriction was limited to the aldosterone fraction.

\section{DISCUSSION}

As early as 1941, Raab (10) reported that an increased blood level of adrenocortical hormones occurs in patients with congestive heart failure. During the next few years very little consideration was given the possible role of the adrenal cortex in the physiology of heart failure. Instead, with the availability of methods for measuring renal hemodynamic function in the intact unanesthetized animal, interest developed in the possible importance of the effect of a reduced rate of glomerular filtration on sodium excretion. In 1950, however, Deming and Luetscher (1) reported the presence of increased sodium-retaining activity in urine from patients with cardiac failure. There followed several studies (11-15) which provided very strong indirect evidence from measurements of electrolytes in sweat, saliva, urine, and 
feces that in patients with cardiac failure or decompensated hepatic cirrhosis adrenocortical hormones promoting sodium retention are present in increased amounts. Also, there were additional reports that increased sodium-retaining activity is present in urine from patients with cardiac failure (3), cirrhosis (2) and nephrosis (4). Since the isolation and crystallization of aldosterone in 1952 , it has been shown that the excretion of aldosterone in urine from patients (16) and from dogs (6) accumulating edema or ascites is increased. Furthermore, a rough inverse correlation of aldosterone output with sodium excretion in urine was described by Luetscher and Johnson (5). The association of increased aldosterone excretion in urine with renal sodium retention was also demonstrated by studies of aldosterone and sodium excretion before and after bilateral adrenalectomy in dogs with caval constriction and ascites (6). During sodium retention in dogs with caval constriction and intact adrenals, aldosterone excretion was elevated above normal, but following bilateral adrenalectomy and discontinuation of hormone therapy aldosterone disappeared from urine and a natriuresis resulted. More quantitative data on the relation of an increased blood level of a sodium-retaining steroid to sodium excretion during ascites formation has been presented previously for adrenalectomized dogs with cardiac failure (17) and for adrenalectomized dogs with caval constriction (15). In both animal preparations it was found that the degree of sodium retention was directly related to the amount of desoxycorticosterone acetate (DCA) administered. Consequently, available evidence indicates a causal relationship between increased circulating aldosterone and sodium retention during cardiac failure and during ascites formation.

It should be pointed out also that increased sodium-retaining hormone alone is insufficient to produce chronic sodium retention in the dog and in some patients with primary aldosteronism (18). Large doses of DCA failed to produce more than transient retention of sodium in normal (19) and in adrenalectomized dogs (20). In the presence of an elevated venous pressure, however, as in the adrenalectomized dog with constriction of the thoracic inferior vena cava (15) or in the adrenalectomized dog with cardiac failure (17), large doses of DCA produced almost complete sodium retention and massive ascites.

The present study demonstrates that aldosterone is secreted in dogs with cardiac failure and in dogs with caval constriction at a rate considerably higher than in normal dogs. Failure to detect aldosterone in peripheral blood although large amounts were present in adrenal vein blood shows that the increased amounts of aldosterone in the adrenal venous effluents resulted from increased secretion and were not representative of the blood level of circulating aldosterone. The observations made during local anesthesia and during blood transfusion provide an estimate of the rate of aldosterone secretion for comparison with urinary aldosterone output. For the data to be meaningful in terms of the average rate of aldosterone secretion during the day, it was necessary to collect blood with the dogs awake; the present data suggest that sodium pentobarbital depressed the rate of aldosterone secretion. Also, Farrell, Rosnagle, and Rauschkolb (21) demonstrated that bleeding stimulates the adrenal cortex to secrete aldosterone. In the present study, a transfusion was given to replace the blood removed and, consequently, to prevent the effect of bleeding per se on aldosterone secretion. If the average value for the rate of aldosterone secretion per hour is used to provide a rough estimate of daily secretion, the rates of aldosterone secretion were $560 \mu \mathrm{g}$. per day for the dogs with caval constriction and $780 \mu \mathrm{g}$. per day for the dogs with heart failure. The rate of excretion of aldosterone in urine from dogs with heart failure and from dogs with constriction of the thoracic inferior vena cava varied from 2 to $6 \mu \mathrm{g}$. per day (6). Similar calculations from the data on normal dogs yield a value of $130 \mu \mathrm{g}$. per day for the rate of aldosterone secretion. No aldosterone was detected in 4 days of normal dog urine but chromatographic fractionation and assay of a 26-day pool of normal dog urine showed that aldosterone was present (6). It appears, therefore, that only 1 per cent or less of secreted aldosterone is excreted in urine from the dog. These findings call attention to the limitation of studies of urinary excretion for evaluation of the factors which regulate the rate of aldosterone secretion.

The sequence of events between the primary myocardial defect in heart failure or the primary 
hepatic defect in cirrhosis of the liver ${ }^{2}$ and the mechanism which stimulates the adrenal cortex to secrete aldosterone has not been clearly delineated. In the dog with caval constriction an elevated venous pressure below the constricting ligature is usually the only detectable alteration in cardiovascular function (22). This finding suggests that elevated venous pressure initiates the sequence of events leading to increased secretion of aldosterone and the subsequent renal retention of sodium. Previous work $(22,23)$ indicated that the influence of venous pressure on the adrenal cortex is indirect; hepatic venous congestion, which is essential to the formation of ascites with a high protein content, appears to be the important consequence of venous hypertension. Whereas sufficient constriction of the thoracic inferior vena cava above the hepatic venous outflow produces ascites consistently (24), constriction of the inferior vena cava above the renal $(24,25)$ or adrenal (23) veins but below the diaphragm or constriction of the hepatic portal vein (23-24) fails to result in chronic sodium retention. The importance of the liver may be related to the permeability of its vascular and lymphatic beds which are areas where fluid and electrolytes escape readily from the blood stream. It is uncertain whether the initial ascites forms before or after a positive sodium balance is established by the kidney but observations have shown that the first alternative is possible. In both dogs with caval constriction and dogs with pulmonic stenosis, the initial formation of ascites was observed in the absence of sodium intake; ascites formed during the first 4 days after caval constriction or pulmonic stenosis while food was withheld (26). The extravasated fluid and electrolytes and resultant ascites formation were associated with increased urinary excretion of aldosterone. The findings from this series of studies are consistent with the hypothesis proposed by Peters several years ago (27) that an elevated venous pressure, extravasation of fluid and electrolytes, adrenocortical stimulation with the consequent increased secretion of a sodiumretaining hormone, and sodium retention occur in this order.

2 The dog with thoracic inferior vena cava constriction and ascites resembles closely the patient with decompensated hepatic cirrhosis in regard to electrolyte metabolism.

\section{SUMMARY AND CONCLUSIONS}

Adrenal vein blood was collected from six normal dogs, three dogs with thoracic inferior vena cava constriction and four dogs with right-sided congestive heart failure. Chromatographic fractionation of the blood and assay of the various fractions showed activity causing sodium retention and increased potassium excretion to be present consistently in only one fraction, the aldosterone fraction. Studies of aldosterone in peripheral and adrenal vein blood from the same animals with either heart failure or thoracic caval constriction demonstrated aldosterone in adrenal venous blood only; it may be concluded, therefore, that aldosterone present in adrenal vein blood represents secreted aldosterone. The rates of aldosterone secretion were significantly greater for dogs with thoracic caval constriction or dogs with heart failure than for normal dogs. From data obtained using local anesthesia, the average rate of aldosterone secretion for normal dogs was $2.7 \mu \mathrm{g}$. per hr., for dogs with caval constriction, $11.6 \mu \mathrm{g}$. per hr., and for dogs with heart failure, 16.5 $\mu \mathrm{g}$. per hr.

\section{ACKNOWLEDGMENTS}

Lala Mathers Dunbar and Audrey Jackson rendered valuable technical assistance.

\section{REFERENCES}

1. Deming, Q. B., and Luetscher, J. A., Jr., Bioassay of desoxycorticosterone-like material in urine. Proc. Soc. Exper. Biol. \& Med., 1950, 73, 171.

2. Chart, J. J., and Shipley, E. S., The mechanism of sodium retention in cirrhosis of the liver. J. Clin. Invest., 1953, 32, 560.

3. Singer, B., and Wener, J., Excretion of sodiumretaining substances in patients with congestive heart failure. Am. Heart J., 1953, 45, 795.

4. McCall, M. F., and Singer, B., Studies in nephrosis: chemical corticoids, salt-retaining factor, and effect of ACTH. J. Clin. Endocrinol. \& Metab., $1953,13,1157$.

5. Luetscher, J. A., Jr., and Johnson, B. B., Observations on the sodium-retaining corticoid (aldosterone) in the urine of children and adults in relation to sodium balance and edema. J. Clin. Invest., 1954, 33, 1441.

6. Davis, J. O., Goodkind, M. J., Pechet, M. M., and Ball, W. C., Jr., Increased excretion of aldosterone in urine from dogs with right-sided congestive 
heart failure and from dogs with thoracic inferior vena cava constriction. Am. J. Physiol., 1956, $187,45$.

7. Davis, J. O., Hyatt, R. E., and Howell, D. S., Rightsided congestive heart failure in dogs produced by controlled progressive constriction of the pulmonary artery. Circ. Research, 1955, 3, 252.

8. Liddle, G. W., Cornfield, J., Casper, A. G. T., and Bartter, F. C., The physiological basis for a method of assaying aldosterone in extracts of human urine. J. Clin. Invest., 1955, 34, 1410.

9. Farrell, G. L., Rauschkolb, E. W., and Royce, P. C., Secretion of aldosterone by the adrenal of the dog. Effects of hypophysectomy and ACTH. Am. J. Physiol., 1955, 182, 269.

10. Raab, W., Adrenocortical compounds in the blood. Relation of their quantity to arterial hypertension, renal insufficiency and congestive heart failure. Arch. Int. Med., 1941, 68, 713.

11. Eisenmenger, W. J., Blondheim, S. H., Bongiovanni, A. M., and Kunkel, H. G., Electrolyte studies on patients with cirrhosis of the liver. J. Clin. Invest., 1950, 29, 1491.

12. White, A. G., Gordon, H., and Leiter, L., Studies in edema. II. The effect of congestive heart failure on saliva electrolyte concentrations. J. Clin. Invest., 1950, 29, 1445.

13. Bongiovanni, A. M., and Eisenmenger, W. J., Adrenal cortical metabolism in chronic liver disease. J. Clin. Endocrinol., 1951, 11, 152.

14. Berger, E. Y., and Steele, J. M., Suppression of sodium excretion by the colon in congestive heart failure and cirrhosis of the liver demonstrated by the use of cation exchange resins. J. Clin. Invest., 1952, 31, 451.

15. Davis, J. O., Howell, D. S., and Southworth, J. L., Mechanisms of fluid and electrolyte retention in experimental preparations in dogs. III. Effect of adrenalectomy and subsequent desoxycorticosterone acetate administration on ascites formation. Circ. Research, 1953, 1, 260.
16. Luetscher, J. A., Jr., Neher, R., and Wettstein, A., Isolation of crystalline aldosterone from the urine of a nephrotic patient. Experientia, 1954, 10, 456.

17. Davis, J. O., Howell, D. S., and Hyatt, R. E., Sodium excretion in adrenalectomized dogs with chronic cardiac failure produced by pulmonary artery constriction. Am. J. Physiol., 1955, 183, 263.

18. Conn, J. W., Presidential Address. II. Primary aldosteronism, a new clinical syndrome. J. Lab. \& Clin. Med., 1955, 45, 6.

19. Davis, J. O., and Howell, D. S., Comparative effect of ACTH, cortisone and DCA on renal function, electrolyte excretion and water exchange in normal dogs. Endocrinology, 1953, 52, 245.

20. Gaudino, M., and Levitt, M. F., Influence of the adrenal cortex on body water distribution and renal function. J. Clin. Invest., 1949, 28, 1487.

21. Farrell, G. L., Rosnagle, R. S., and Rauschkolb, E. W., Increased aldosterone secretion in response to blood loss. Circ. Research, 1956, 4, 606.

22. Davis, J. O., and Howell, D. S., Mechanisms of fluid and electrolyte retention in experimental preparations in dogs. II. With thoracic inferior vena cava constriction. Circ. Research, 1953, 1, 171.

23. Davis, J. O., and Howell, D. S., Unpublished data.

24. Volwiler, W., Grindlay, J. H., and Bollman, J. L., The relation of portal vein pressure to the formation of ascites-an experimental study. Gastroenterology, 1950, 14, 40.

25. Hwang, W., Akman, L. C., Miller, A. J., Silber, E. N., Stamler, J., and Katz, L. N., Effects of sustained elevation of renal venous pressure on sodium excretion in unanesthetized dog. Am. J. Physiol., 1950, 162, 649.

26. Ball, W. C., Jr., Davis, J. O., and Goodkind, M. J., Ascites formation without sodium retention in dogs with thoracic inferior vena cava constriction and dogs with pulmonary artery constriction. Federation Proc., 1956, 15, 7.

27. Peters, J. P., The problem of cardiac edema. Am. J. Med., 1952, 12, 66. 